\title{
Experimental Neurobiology: The Past, Present, and Future
}

\author{
Young Jun $\mathrm{Oh}^{1}$, Pyung-Lim $\mathrm{Han}^{2}$ and C. Justin Lee ${ }^{3 *}$ \\ ${ }^{1}$ Yonsei University, Seoul 03722, ${ }^{2}$ Ewha Womans University, Seoul 03760, ${ }^{3}$ Insitute for Basic Science, \\ Center for Cognition and Sociality, Daejeon 34125, Korea
}

The first issue of Experimental Neurobiology (EN) was published in December of 1992 as the official journal of Korean Society for Neurobiology. While a domestic journal, EN was unique in that all manuscripts submitted had to be in English. The late Professor Joseph Jin Chang at Sogang University was the most prominent founder of the journal and almost single-handedly managed the publication of EN for the subsequent six years. With his commitment, passion and most importantly leadership, EN successfully started the voyage as a journal covering interdisciplinary investigations in all fields of neuroscience.

In 1997, Korean Society for Neurobiology and Korean Society for Neuroscience were merged to become Korean Society for Brain and Neural Science (KSBNS) to encompass broad aspects of neuroscience, as a representative organization for scientists and physicians devoted to understanding of the brain and the nervous systems. In 1998, EN became the official journal of KSBNS. From 2000 to 2008, EN invited several influential figures to serve as Editor-in-Chief (Table 1), Managing Editor and Reviewing Editor. During this period, Managing Editor was in full charge of handling all the processes of publishing articles. The continuous success of the journal was directly related to the hard work and devotion of all the editorial members, and we were very fortunate to have four extraordinary Managing Editors, and we are thrilled to acknowledge them for their invaluable dedication and service: Professors Onyou Hwang (University of Ulsan, 2000 2002), Yunhee Kim-Kwon (Kyung Hee University, 2003 2004), Suja Oh (The Catholic University of Korea, 2005 2006) and Insop Shim (Kyung Hee University, 2006 2008).

\footnotetext{
* To whom correspondence should be addressed. TEL: 82-42-878-9150, FAX: 82-42-878-9151 e-mail:cjl@ibs.re.kr
}

From 2008, dramatic changes were made in order to further upgrade the quality of EN and to cultivate a solid platform to be recognized as a global journal. First, Editor-in-Chief rather than Managing Editor, took the charge of managing publication of each issue. Starting from 2009, EN was subdivided into several sections and consequently invited Associate Editor and Editorial Board Members for each section. These sections included Cellular and Molecular Neuroscience, Development/Differentiation/Regeneration Neuroscience, Neurobiology of Diseases, System/Cognitive/ Behavioral Neuroscience, Neuromodeling/Neuroimaging/Neuroengineering, Clinical Neuroscience, and Methods and Protocol. In addition, EN started to publish not only original papers but also review articles on a regular basis. EN also invited distinguished foreign neuroscientists as Editorial Board Members (USA, 12; Japan, 4; China, 3; Germany, 2; Hong Kong, 2; England, 1; Australia, 1; Israel, 1). In 2010, EN was approved as a candidate registered journal by the Korean Research Foundation, following the previous year's unsuccessful attempt, and became listed in the Korean Citation Index.

Table 1. Chronicles of Editor-in-Chiefs of EN

\begin{tabular}{|c|c|c|}
\hline Year & Editor-in-Chief & Affiliation \\
\hline $1992 \sim 1999$ & Joseph Jin Chang & Sogang University \\
\hline $2000 \sim 2002$ & Jun Kim & $\begin{array}{l}\text { Seoul National University } \\
\text { College of Medicine }\end{array}$ \\
\hline $2003 \sim 2004$ & Dong Goo Kim & $\begin{array}{l}\text { Yonsei University College of } \\
\text { Medicine }\end{array}$ \\
\hline $2005 \sim 2006$ & Myung-Hoon Chun & $\begin{array}{l}\text { The Catholic University of Korea } \\
\text { College of Medicine }\end{array}$ \\
\hline 2007 & Kyung Ah Park & $\begin{array}{l}\text { Yonsei University College of } \\
\text { Medicine }\end{array}$ \\
\hline 2008 2012 & Young Jun Oh & Yonsei University \\
\hline 2013 2016 & Pyung-Lim Han & Ewha Womans University \\
\hline $2017 \sim 2020$ & C. Justin Lee & Institute for Basic Science \\
\hline
\end{tabular}

Copyright $\odot$ Experimental Neurobiology 2019. www.enjournal.org
This is an Open Access article distributed under the terms of the Creative Commons Attribution Non-Commercial License (http://creativecommons.org/licenses/by-nc/4.0) which permits unrestricted non-commercial use, distribution, and reproduction in any medium, provided the original work is properly cited. 
The most dramatic and memorable changes in the EN history would be the ambitious effort to establish an e-journal system. To do so, being the Editor-in-Chief at the time (2008 2012), Professor Young Jun Oh (Yonsei University) initiated formation of the Publication Committee. Obviously, the crucial mission of the Committee was to establish the journal with a brand new format, and most importantly, an on-line reviewing system that would attract interests from all neuroscience fields all over the world. Starting from 2011, EN in the new format has been publishing 4 issues per year. Archives of full-text articles are now available from Volume 20, number 2 issue. As a consequence, EN successfully signed an memorandum of understanding $(\mathrm{MoU})$ with and became indexed in PubMed on November $23^{\text {rd }}, 2011$. All these accomplishments would not have been possible without the time, effort, dedication, innovative initiatives, and positive spirits of the eight members of the Publication Committee that go beyond and above what one can imagine. They are Professors Jong Eun Lee (Yonsei University), Onyou Hwang (University of Ulsan), Pyung-Lim Han (Ewha Womans University), Jaesang Kim (Ewha Womans University), Hyeon Son (Hanyang University), Kwang Chul Chung (Yonsei University), Soochul Park (Sookmyoung Women's University) and Young Jun Oh. Special gratitude also goes to Professor Kyungjin Kim (DGIST), who at the time was the head of the $21^{\text {st }}$ Century Brain Frontier Project, for his kind financial and moral support. As described below, EN's bold attempt has continued under the management by consecutive two brilliant Editor-in-Chiefs; Professor Pyung-Lim Han and Dr. C. Justin Lee (Institute for Basic Science).

Professor Pyung-Lim Han (Ewha Womans University) was appointed to serve as the Editor-in-Chief of EN from 2013 to 2016. The new EN editorial board consisted of the following members in the specific titles/sections: Executive Editors (Professor Jong Eun Lee, Yonsei University; Professor Jung-Joon Sung, Seoul National University), Managing Editors (Professor HyoungChun Kim, Kangwon National University; Professor Soochul Park, Sookmyung Women's University; Professor Seung-Jae Lee, Seoul National University), and 26 Associate Editors who were in charge of Cellular and Molecular Neuroscience, Neurobiology of Diseases, Clinical Neuroscience, Development/Differentiation/ Regeneration Neuroscience, or Systems/cognitive/behavioral Neuroscience. In the meantime, EN frequently invited excellent guest editors to organize manuscripts with expertise in a special topic (Professor Eunhye Joe, Ajou University; Professor KyoungHo Suk, Kyungpook National University; Professor Seung-Jae Lee, Seoul National University; Professor Chan Young Shin, Konkuk University; Professor Jung-Joon Sung, Seoul National University).

One long-standing mission of EN to resolve was the establishment of a stably running system to publish high quality papers in a reasonable volume. After considering the realistic environment in 2013, the EN editorial team decided to set the following specific objectives: (i) doubling the publishing volume to 40 and more articles each year, and (ii) putting all possible energies to obtain the SCOPUS and SCIE titles as soon as possible. In the beginning of 2013, the Korean Society for Neurodegenerative Disease (KSND) was about to launch its own official journal. That news was somewhat challenging to EN, since potential scientists who might contribute good manuscripts to EN were not many, but could be split by this activity. Fortunately, EN found a solution to serve as an official journal for both KSBNS and KSND. In fact, EN had received many generous supports from both KSBNS and KSND in the successful publication of total 162 manuscripts during the period of 2013 2016. Furthermore, from $2016 \mathrm{EN}$ started to publish six volumes a year, thus shaping a bimonthly journal.

Continuing the effects to increase the publication volume and the publication frequency, and to seek for more qualified manuscripts, EN had been successfully included in the list of KCI (Korea Citation Index) journals in 2015, in the SCOPUS journals in 2016, and finally the SCIE journal titles in 2017. The manuscripts published by EN were read by many neuroscientists in the world more than that many people thought. For example, EN published 82 articles in the two consecutive years of 2014 2015, and those EN articles were cited in 2016 by 305 peer-reviewed papers published in other journals. Further analysis indicated that the corresponding authors who cited the 2014 2015 EN papers in 2016 were working in USA (62), China (54), Korea (40), Italy (14), Canada (10), Spain (10), Austria (9), German (9), Japan (9), Iran (7), India (6), Netherlands (6), Egypt (5), Brazil (5), England (4), Switzerland (4), Portugal (4), Mexico (4), Poland (3), France (3), Taipei (3), Pakistan (3), Belgium (3), and other 19 countries (305 citations by scientists from total 43 countries). Thus, overall, $86.9 \%$ of 305 citations were made by foreign scientists, whereas only $13.1 \%$ were contributed by Korean scientists. All those were about what happened in 2016, the year before EN received the SCIE journal title. These data imply that the manuscripts published by EN were reasonably qualified, and were stayed in the areas where many scientists are currently working. Our statistical analysis indicated that EN's impact factors was 2.98 in 2014, 3.29 in 2015, 3.72 in 2016, and now officially 3.81 in 2017. All these were possible because the Korean neuroscience research community had worked together and had a strong will and goal to advance EN to be an international journal leading the cutting-edge neuroscience. These achievements may represent the current status and activities of the neuroscience community in Korea and presents many hopes and new missions to lead neuroscience in the future.

The rich history and many hard-working editorial and manage- 
rial staff of the past and present make the future of EN as bright as the full moon in Chuseok. Since the succeeding editor-in-chief, Dr. C. Justin Lee (Institute for Basic Science), has been appointed in the beginning of 2017, the journal is now headed towards achieving the impact factor of 5 within 3 or 4 years. To achieve this goal, EN has started with three distinctive visions. Firstly, EN is now focusing on enhancing the scientific quality of the published papers by restructuring the editorial board. EN has appointed two deputy editors and eight executive editors (section editors) for each section (Table 2). The two deputy editors in collaboration with the chief editor initially screen the incoming manuscripts and make decisions on whether to proceed with a peer review or not. Each manuscript which has passed the initial screening is then assigned to a relevant section editor to be handled for a peer review process. Each handling editor then invites potential reviewers, mostly from the associate editors, who are well-established, scientifically-active researchers in each section. Implementation of this new review process ensures a high quality of review, as the handling editors are asked to strengthen the review process by requesting authors to perform additional experiments with $2^{\text {nd }}$ and $3^{\text {rd }}$ rounds of revisions. EN has also added new sections of Advanced Neurotechnology and Negative Data and Reproducibility sections to incorporate the field of fast-advancing neuro-tools and neuro-devices, as well as to embrace the increasing number of studies that show profoundly different or opposite results as the previously published papers from other journals. To speed up the processing of the manuscript for review, EN now gives an option for authors to choose either the Quick Processing or Regular Processing. Quick Processing should take less than a month for review processing from submission to acceptance (not including authors' revision period) and final online publication in the Forthcoming or as the final publication at EN home page.

Secondly, year 2017 marks the year of drastic change for EN by taking an initiative to charge a nominal handling and publication fee for each accepted manuscript before publication. This drastic initiative was made in order to achieve EN's financial independence from the two societies, KSBNS and KSND as a long-term goal. The most prominent consequence of this initiative was the fact that the total number of submissions of low quality manuscripts from developing countries were dramatically decreased. After the initiative, EN now enjoys submissions of mostly high quality manuscripts. In addition to the implementation of a handling and publication fee, EN has implemented an honorarium system for the reviewers. EN now pays a nominal amount of honorarium for each of two or three reviewers and the handling editor for each manuscript that has gone into peer-review. The purpose of this honorarium system was to ensure the high quality of reviews by compensating the efforts put forth by each reviewer and handling editor. The obvious consequence of this implementation of honorarium system has been that each reviewer now takes each review seriously and puts full efforts to make scientific and constructive comments and criticisms. With these drastic changes, the review process has been more rigorous and the quality of papers has been enhanced. Furthermore, since the release of SCIE impact factor in 2017, the number of high quality submissions has increased and subsequent number of publications per year has steadily increased to reach about 10 papers per bi-monthly issue, or 60 papers per year. This certainly increases the inflow of handling and publication fees for each accepted manuscript. In addition, EN will completely renew the journal home page in September 2019 and add new features, including banner advertisements to collect additional fees. Taken together, all of these factors should contribute to the future financial independence of EN.

Finally, EN is now planning to launch a sister premier journal dedicated to brain science in near future. Motivated by the increasing quality and number of publications, the time is ripe for a premier journal suitable for publishing high impact papers. If the goal of $\mathrm{EN}$ is to reach the impact factor of 5 , the goal of this premier sister journal would be an impact factor above 10. This plan for a premier sister journal timely reflects the need for a premier

Table 2. Editorial staff of EN 2019

\begin{tabular}{|c|c|c|}
\hline Editor-in-Chief & C. Justin Lee & Institute for Basic Science \\
\hline Deputy Editor & Jong Eun Lee & Yonsei University College of Medicine, Korea \\
\hline Deputy Editor & Byung-Ok Choi & Sungkyunkwan University School of Medicine, Korea \\
\hline \multicolumn{3}{|l|}{ Executive Editors (Section Editors) } \\
\hline Molecular and Cellular Neuroscience: & Sang Jeong Kim & Seoul National University, Korea \\
\hline Development/Differentiation/Regeneration: & Woong Sun & Korea University College of Medicine, Korea \\
\hline Neurobiology of Diseases: & Byung Gon Kim & Ajou University School of Medicine, Korea \\
\hline Glial Biology: & Eun-hye Joe & Ajou University School of Medicine, Korea \\
\hline Cinical Neuroscience: & In Kyoon Lyoo & Ewha Womans University, Korea \\
\hline Advanced Neurotechnology \& Neuroengineering: & Yoonkey Nam & Korea Advanced Institute of Science and Technology, Korea \\
\hline Negative Data and Reproducibility in Neuroscience: & Shawn Hyunsoo Je & Duke-NUS, Singapore \\
\hline
\end{tabular}


brain research journal for the fast advancing brain research in Asia, especially in Korea, Japan, and China. EN has gained sufficient expertise and experience to establish such a prestigious journal, thanks to the dedication and efforts by previous editor-in-chiefs, managing editors, editorial staffs, editorial managers. With these past advancements, present enhancements, and future plans, the future of EN is bright as a shining star. 